\title{
NUMERICAL INVESTIGATION AND APPLICATION OF FRACTIONAL DYNAMICAL SYSTEMS
}

\author{
LIBO FENG ${ }^{\circ}$
}

(Received 8 September 2019; first published online 23 October 2019)

2010 Mathematics subject classification: primary 26A33; secondary 65N06, 65N08, 65N30, 65N12.

Keywords and phrases: fractional diffusion equation, finite element method, control volume method, irregular convex domain, unstructured mesh, fluid models, stability and convergence analysis.

The use of fractional differential equations (FDEs) to model the nonlocality, memory, spatial heterogeneity and anomalous diffusion inherent in many real-world problems has attracted considerable attention in many fields of science including physics, electrical systems, bioengineering, hydrology, chemistry, biochemistry and finance. FDEs help in the computational modelling of these complex systems by interpolating between the integer orders of differential equations to capture nonlocal relations in time and space using power-law memory kernels. This growing interest has led to an intense worldwide research focus to uncover new theoretical and numerical methods for solving fractional dynamical systems.

An important example of a fractional dynamical system arises in the study of non-Newtonian fluids where the heterogeneity of the internal temperature and the distribution of fluid density cause the transport in a viscoelastic non-Newtonian fluid to become 'anisotropic' when an external force by shear flow is applied. The classical research methods of universal application and mathematical characterisation used in studying Newtonian fluids are no longer applicable because non-Newtonian fluids involve complex multiterm time fractional dynamical systems with both diffusion and time fractional diffusion terms, as well as a nonlinear reaction term. In addition, many complex fractional dynamical models involve a Riesz fractional operator and, typically, must be solved over irregular domains, for example the time-space fractional Bloch-Torrey equation, which can be applied to fit the magnetic resonance imaging diffusion images obtained from microenvironments such as human brain tissue and human articular cartilage.

Thesis submitted to Queensland University of Technology in October 2018; degree approved on 28 February 2019; supervisors Fawang Liu, Ian Turner and Qianqian Yang.

(C) 2019 Australian Mathematical Publishing Association Inc. 
The aim of this thesis is to develop new computational fractional dynamical models for key application areas of science and engineering and solve them using novel numerical methods. This overall aim will include the following three main objectives:

- to develop new numerical methods and analytical techniques for simulating complex fractional dynamical models to reduce computational cost, which is achieved by utilising high-order numerical methods and fast algorithms;

- to develop numerical methods for complex fractional dynamical models with the Riesz fractional operator on irregular domains, which is achieved by using the finite element method and finite volume method combining the unstructured mesh capability to approximate the space fractional derivative;

- $\quad$ to develop numerical methods for viscoelastic non-Newtonian fluid models, such as the generalised Maxwell fluid model, the generalised Oldroyd-B fluid model and the generalised Burgers' fluid model, which is achieved by applying mixed difference schemes to discretise the different time fractional derivatives.

One original contribution of this thesis is the treatment of the Riesz space fractional derivative on irregular convex domains. Based on the Galerkin finite element method (FEM) with an unstructured mesh, a novel numerical technique to treat the Riesz space fractional derivative on irregular convex and nonconvex domains is developed and the theoretical analysis is presented, which is more flexible than the finite difference method. In addition, a novel unstructured mesh control volume method to calculate the space fractional derivative on irregular convex domains is developed. It can reduce CPU time significantly while retaining the same accuracy and approximation property as the unstructured mesh finite element method.

Another important contribution of this thesis is that a unified numerical scheme to solve a class of novel multiterm time fractional diffusion-wave and subdiffusion equations is presented and the rigorous stability and convergence analysis is established, which not only can be extended to solve the generalised Maxwell fluid model, the generalised Oldroyd-B fluid model and the generalised Burgers' fluid model but also to solve the general multiterm time fractional diffusion-wave or subdiffusion equation, the time-fractional telegraph equation, the fractional cable equation and the fractional Cattaneo diffusion equation.

A series of papers arising from this thesis have been published. These papers address a fast second-order accurate method for a two-sided space-fractional diffusion equation with variable coefficients [6], the unstructured mesh finite element method for the two-dimensional time-space Riesz fractional diffusion equation on irregular convex domains [3], the unstructured mesh control volume method for twodimensional space fractional diffusion equations with variable coefficients on convex domains [2], the finite difference method for the generalised Oldroyd-B fluid [4], magnetohydrodynamic Couette flow of a generalised Oldroyd-B fluid [5] and the finite element method for a novel two-dimensional multiterm time-fractional mixed subdiffusion and diffusion-wave equation on convex domains [1]. There are also 
two papers on the novel finite volume method for the Riesz space distributed-order diffusion equation [7] and for the advection-diffusion equation [8].

\section{References}

[1] L. Feng, F. Liu and I. Turner, 'Finite difference/finite element method for a novel 2D multi-term time-fractional mixed sub-diffusion and diffusion-wave equation on convex domains', Commun. Nonlinear Sci. Numer. Simul. 70 (2019), 354-371.

[2] L. Feng, F. Liu and I. Turner, 'An unstructured mesh control volume method for two-dimensional space fractional diffusion equations with variable coefficients on convex domains', J. Comput. Appl. Math. 364 (2020), 112319.

[3] L. Feng, F. Liu, I. Turner, Q. Yang and P. Zhuang, 'Unstructured mesh finite difference/finite element method for the 2D time-space Riesz fractional diffusion equation on irregular convex domains', Appl. Math. Model. 59 (2018), 441-463.

[4] L. Feng, F. Liu, I. Turner and P. Zhuang, 'Numerical methods and analysis for simulating the flow of a generalized Oldroyd-B fluid between two infinite parallel rigid plates', Intl J. Heat Mass Transfer 115 (2017), 1309-1320.

[5] L. Feng, F. Liu, I. Turner and P. Zhuang, 'Novel numerical analysis of multi-term time fractional viscoelastic non-Newtonian fluid models for simulating unsteady MHD Couette flow of a generalized Oldroyd-B fluid', Fract. Calc. Appl. Anal. 21(4) (2018), 1073-1103.

[6] L. Feng, P. Zhuang, F. Liu, I. Turner, V. Anh and J. Li, 'A fast second-order accurate method for a two-sided space-fractional diffusion equation with variable coefficients', Comput. Math. Appl. $\mathbf{7 3}$ (2017), 1155-1171.

[7] J. Li, F. Liu, L. Feng and I. Turner, 'A novel finite volume method for the Riesz space distributedorder diffusion equation', Comput. Math. Appl. 74 (2017), 772-783.

[8] J. Li, F. Liu, L. Feng and I. Turner, 'A novel finite volume method for the Riesz space distributedorder advection-diffusion equation', Appl. Math. Model. 46 (2017), 536-553.

LIBO FENG, School of Mathematical Sciences,

Queensland University of Technology, GPO Box 2434,

Brisbane, Queensland 4001, Australia

e-mail: fenglibo2012@126.com 